\title{
Surface Characterization and Interfacial Adhesion in MEMS Devices
}

\author{
Y. F. Peng and Y. B. Guo \\ Xiamen University \\ China
}

\section{Introduction}

The characteristic size of MEMS is ranging from atomic and molecular scales to micrometer and several millimeters scales. Components that reach micro-scale size have a high surface to volume ratio, which leaves them be highly subjected to micro-scale effect and susceptible to surface forces. Devices that utilize MEMS technology will often having mating surfaces. Adhesion force can arise from any number of phenomenon such as van der Waals, capillary, ionic and molecular forces. The components used in MEMS structures are very light (on the order of a few micrograms) and operate under very light loads (on the order of a few micrograms to a few milligrams). Surface forces between the adjacent surfaces are becoming dominant over the inertial force in MEMS devices. Because of the micro-sized component, the adhesion forces can pull the adjacent compliant structure into contact and result the interfacial adhesion, which may cause the device-malfunction to a great extent. The operation and performance of lightly loaded micro/nano components in MEMS are highly dependent on the adhesive interactions between mating surfaces. In a word, it is important that the mechanisms of interfacial adhesion should be explained, and separating techniques should be added to the design of MEMS scale components to ensure there is no unwanted contact. Furthermore, the interfacial adhesion between two adjacent mating surfaces is determined by the interaction of rough surfaces. The surface is all rough though in different range. The interactions among different asperities are complicated because the surface topography is consisting of so many asperities. It stands to reason that the proper surface characterization is necessary to elucidate the interfacial adhesion.

The interfacial adhesion is the science and technology of two interacting surfaces in relative motion and of related subjects and practices. It is also valuable in the fundamental understanding of interfacial phenomena to provide a bridge between science and engineering in MEMS. The differences between the conventional or macro-contact and micro/nano-adhesion are contrasted in Table 1. In macro-contact mechanics, tests are conducted on components with relatively large mass under heavily loaded conditions. In these tests, contacting between mating surfaces is inevitable and the bulk properties of mating components dominate the contacting performance. In micro/nano-adhesion, measurements are made on components, at least one of the mating components, with relatively small mass under lightly loaded conditions. The interaction is not limited only to the contacting condition. In this situation, though without contact, the attractive interaction 
between mating opposite surfaces at small approaching distance can't be neglected. Some of the smaller asperities on the micro-sized surface will be stretched, while some of the taller ones will be compressed through contact. The classical contact mechanics is no longer valid in analyzing the interaction of mating micro/nano-sized surface. It is necessary to explore methods to solve the interfacial adhesion problems in MEMS devices.

\begin{tabular}{|l|l|l|l|}
\hline \multicolumn{2}{|l|}{ Macro-contact mechanics } & \multicolumn{2}{|l|}{ Micro/Nano adhesion } \\
\hline \multirow{3}{*}{ Condition } & Contact (Inevitable) & \multirow{3}{*}{ Condition } & Contact or non-contact \\
\cline { 2 - 2 } & Large mass & Small mass \\
\cline { 2 - 2 } \cline { 4 - 4 } Method & Heavy load & Mertz theory & $\begin{array}{l}\text { Light or zero load } \\
\text { surface forces }\end{array}$ \\
\cline { 2 - 2 } Target & Linear elastic mechanics & Bulk material & $\begin{array}{l}\text { Surface (Few atomic layers to } \\
\text { several } \mu \mathrm{m} \text { depths) }\end{array}$ \\
\hline
\end{tabular}

Table 1. Comparison between macro-contact and micro/nano-adhesion

In this chapter, we will take a close look at surface geometric structure, or surface topography, and surface forces to elucidate the adhesion problems between mating microsized MEMS surfaces. Firstly, the complexities of the surface microstructure devices are discussed. Secondly, several typical surface-measurement instruments are introduced. Thirdly, the techniques to characterize the complex micro-scale surfaces are presented. Finally, the surface forces are described in a summary form, and then the adhesion models are given to interpret the adhesive interaction of MEMS devices.

\section{Characterization and modeling of microstructure surface}

\subsection{Complexities of surface microstructure}

Whether a surface is rough or smooth, the answer is - it depends on a roughness sensors used (Bushan, 1999)! The problem of scale-dependent roughness is very intrinsic to solid surfaces. For most solid surfaces it is observed that under repeated magnification, more and more roughness keeps appearing until the atomic scales are reached where roughness occurs in the form of atomic steps (Williams \& Bartlet, 1991) as shown graphically in Fig.1. The roughness often appears random and disordered, ranges from around $10^{-4} \mathrm{~m}(0.1 \mathrm{~mm})$ to about $10^{-9} \mathrm{~m}(1 \mathrm{~nm})$ and does not seem to follow any particular structural pattern (Thomas, 1982). The randomness and the multiple roughness scales both contribute to the complexity of the surface geometric structure.

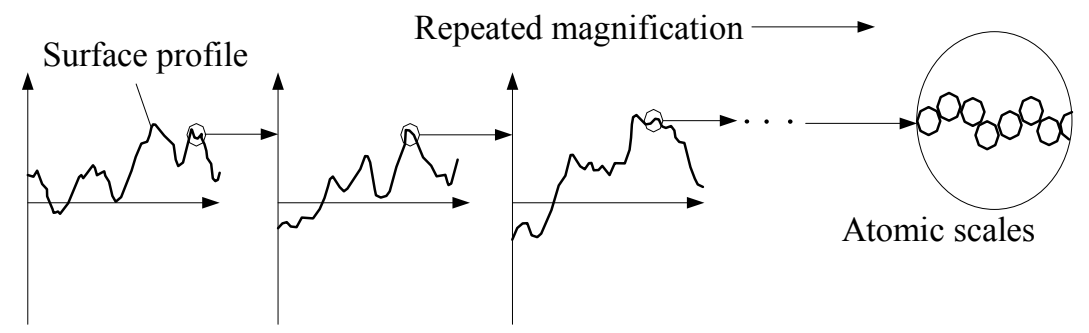

Fig. 1. Scale-dependent of surface roughness (Bhushan, 1999). 


\subsection{Surface measurement techniques}

Because of the complexities of surface microstructure, the measuring techniques and instruments are important to achieve the surface information to characterize and model the surface microstructure in MEMS devices. The accuracy of traditional contact (probing) as well as noncontact techniques has been perfected to a level allowing measurement of roughness in the nanometer range (Fig.2). The most accurate profilometer probes allow measurement of summit heights of several Angstroms (Bennet \& Dancy, 1981; Bhushan et al., 1988). Yet, the comparatively poor lateral (horizontal) resolution significantly limits application of these techniques to the nanometer topographies when the distance between asperities is much less than the solution or $0.1-1 \mu \mathrm{m}$. The development of techniques using probes smaller than the radius of the probing needle or the light wavelength makes it possible to extend the spectrum of surfaces studied (Myshkin et al., 2003). The scanning electron microscope (SEM) technique can be used to gauge topography with a comparable resolution both vertically and laterally by interpreting the emission intensity of the secondary electrons the topographic pattern (Myshkin et al., 1992). The scanning tunneling microscope (STM) has a still finer probe, which is the electron flux tunneled between the target surface and the needle tip. In this case the surface topography resolution is 0.01 and $0.1 \mathrm{~nm}$ in the vertical and lateral directions, respectively (Binnig \& Rohrer, 1982). Hence, the STM technique and others resulting from its progress make it possible to use this approach for more accurate topographic investigations of solids on the nanoscale. Significant prospects are connected with the application of atomic force microscope (AFM) (Sarid, 1991) in which atomic-molecular surface effects are registered.

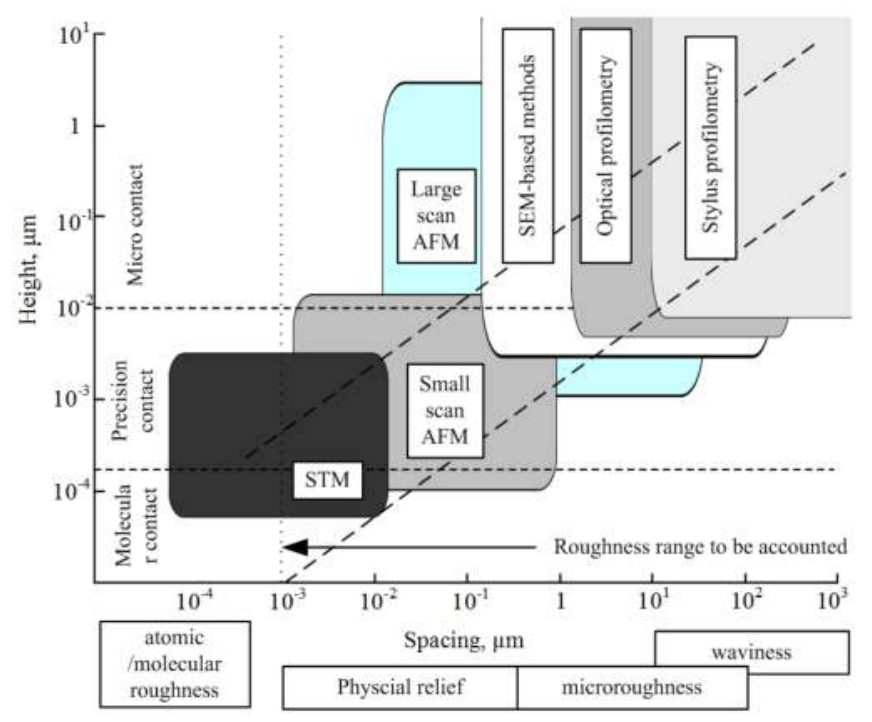

Fig. 2. Diagram of the height and spacing parameters and ranges of vertical-lateral resolution for different methods of roughness measurement (Myshkin et al., 2003).

The scanning tunneling microscope (STM) developed by Dr. Gerd Binnig and Heinrich Rohrer has revolutionized the study of surfaces and is rapidly becoming a required tool in 
almost every surface characterization laboratory. It is the first instrument capable of directly obtaining three-dimensional images of solid surfaces with atomic resolution (Binnig et al., 1982). Today's STMs can be used in the ambient environment for atomic-scale imaging of surfaces. Generally, samples to be imaged with STM must be conductive enough to allow a few nanoamperes of current to flow from the bias voltage source to the area to be scanned. In many cases, nonconductive samples can be coated with a thin layer of a conductive material to facilitate imaging. AFM can be used for measurement of all engineering surfaces which may be either electrically conducting or insulating. AFM has now become a main surface profiler for topographic measurements on micro- to nanoscale (Bhushan \& Blackman, 1991; Oden et al., 1992; Bhushan et al, 1997). STMs, AFMs, and their modifications can be used at extreme magnifications ranging from $10^{-3}$ to $10^{-9} \times$ in $x_{--}, y^{-}$, and $\mathrm{z}$-directions for imaging macro- to atomic dimensions with high-resolution information and for spectroscopy (Bushan, 1999). These instruments can be used in any sample environment such as ambient air (Binnig \& Smith, 1986), various gases (Burnham et al., 1990), liquid (Marti et al., 1987; Binggeli et al., 1993), vacuum (Binnig et al., 1982), low temperatures (Hug et al., 1993), and high temperatures. To decrease the wear of brittle tip and extend its application in biological research, the carbon nanotube (CNT) has been used to probe the sample instead by adhered it on top of a tip (Fang et al., 2008) in AFM. The resolution ratio can reach about $3 \mathrm{~nm}$ with functional single-walled CNT in scanning the grease double molecular membranes, while it is about 15nm for conventional Si and of Si3N4 (Yamachika et al., 2004). Fig.3 shows such a tip-CNT probe and the captured image.
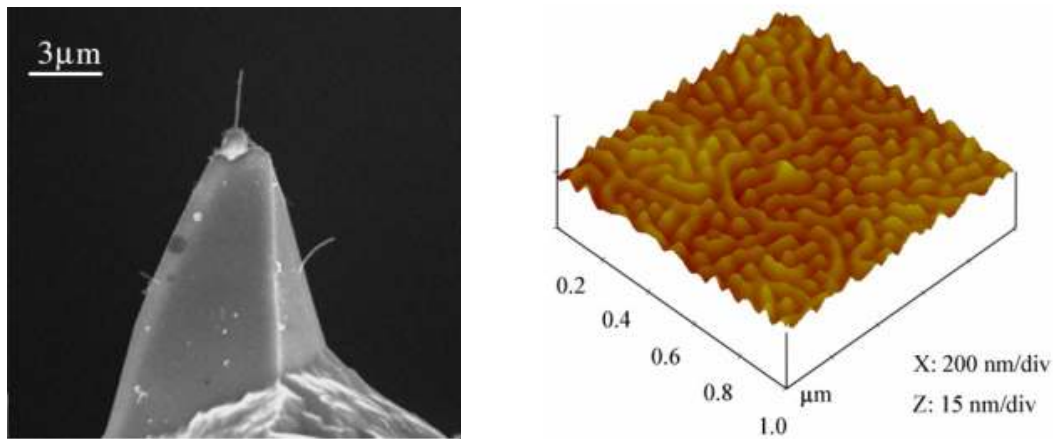

Fig. 3. AFM CNT probe and captured image (Fang et al., 2008); (a). SEM images of CNT probe (b). Images of a styrene-ethylene/butylene-styrene copolymer

\subsection{Surface characterization techniques}

The characterization of the surface roughness on the micro/nanoscale needs more thorough investigation. This is essential for solving interfacial adhesion phenomena. The randomness suggests that the statistical methods of roughness characterization should be adopted to determine the average dimensions of topographical elements forming the surfaces of solids. In addition, a rough surface involves so many length scales ranges from atomic/molecular level to nano or micro scale, then the characterization techniques must be independent of any length scale. In this section, both the statistical and fractal method to characterize surface roughness are presented in a way that is suitable to model adhesion of mating MEMS devices. 


\subsubsection{Probability height distribution}

In "Handbook of Mirco/Nano Tribology", Bhushan has summarized various theories of probability distribution of rough surface (Bhushan, 1999). One of the characteristics of a rough surface is the probability distribution (Papoulis, 1965). It is often found that the normal or Gaussian distribution fits the experimentally obtained probability distribution quite well (Thomas, 1982; Bhushan, 1990). In addition, it is simple to use for mathematical calculation (Greenwood \& Williamson, 1966; Chang et al., 1987). The bell-shaped normal distribution (Papoulis, 1965) which has a variance of unity is given as

$$
g(\bar{z})=\frac{1}{\sqrt{2 \pi}} \exp \left[-\frac{\left(\bar{z}-\bar{z}_{m}\right)^{2}}{2}\right] \quad-\infty<\bar{z}<\infty
$$

where $\bar{z}_{m}=\bar{z} / \sigma$ is the nondimensional mean height, $\sigma$ is the standard deviation. The mean height and the standard deviation can be found from a roughness measurement $z(x, y)$ as

$$
\begin{gathered}
z_{m}=\frac{1}{L_{x} L_{y}} \int_{0}^{L_{x}} \int_{0}^{L_{y}} z(x, y) d x d y=\frac{1}{N_{x} N_{y}} \sum_{i=1}^{N_{x}} \sum_{j=1}^{N_{y}} z\left(x_{i}, y_{j}\right) \\
\sigma=\sqrt{\frac{1}{L_{x} L_{y}} \int_{0}^{L_{x}} \int_{0}^{L_{y}}\left[z(x, y)-z_{m}\right]^{2} d x d y}=\sqrt{\frac{1}{N_{x} N_{y}} \sum_{i=1}^{N_{x}} \sum_{j=1}^{N_{y}}\left[z\left(x_{i}, y_{j}\right)-z_{m}\right]^{2}}
\end{gathered}
$$

Here, $L_{x}$ and $L_{y}$ are the lengths of surface sample, whereas $N_{x}$ and $N_{y}$ are the number of points in the $x$ and $y$ lateral directions, respectively. The integral formulation is for theoretical calculations, whereas the summation is used for calculating the values from finite experimental data.

Although used extensively, the normal distribution has limitations in its applicability. The normal distribution near the tail is not an accurate representation of real surfaces. This is an important point since it is usually the tail of the distribution that is significant for calculating the real area of contact (Bushan, 1999). The inverted chi-squared (ICS) distribution fit the experimental data much better near the tail of the distribution (Brown \& Scholz, 1985). This is given for zero mean and in terms of nondimensional height, $\bar{z}$, as

$$
g(\bar{z})=\frac{(v / 2)^{v / 4}}{\Gamma(v / 2)}\left(\bar{z}_{\max }-\bar{z}\right)^{(v / 2)-1} e\left(\bar{z}-\bar{z}_{\max }\right) \sqrt{v / 2} \quad-\infty<\bar{z}<\bar{z}_{\max }
$$

which has a variance of $2 v$ and a maximum height $\bar{z}_{\max }=\sqrt{v / 2}$. The advantage of the ICS distribution is it has a finite maximum height, as does a real surface, and has a controlling parameter $v$, which gives a better fit to the topography data. It is found that as $v$ increases, the ICS distribution tends toward the normal distribution (Bushan, 1999; Brown \& Scholz, 1985).

Berry and Hannay (Berry \& Hannay, 1978) suggested that the variance can be represented as follows:

$$
\sigma^{2} \approx L^{n}
$$

where $L$ is the length of the sample and $n$ varies between 0 and 2 . 
If the exponent $n$ in Equation (5) is equal to zero, then the rough surface is generally to be a statically stationary process. This means that the measured roughness sample is a true statistical representation of the entire rough surface. However, $n$ is not equal to zero in the general cases. Then a rough surface is assumed to be a nonstationary random process and the standard deviation is scale dependent, which arises from the probability distribution of a small surface region may be different from that of the larger one. The gathered roughness measurements of a wide range surfaces by Sayles and Thomas (Sayles \& Thomas, 1978) have shown that the variance of the height distribution is a function of the sample length and in fact suggested that the variance varied as $\sigma^{2} \approx L$. This behavior implies that the surface is a nonstationary process and any length of the surface cannot fully represent the surface in a statistical sense.

Other statistical parameters, such as rms slope $\sigma^{\prime}$ and rms curvature $\sigma^{\prime \prime}$ proposed by Nayak (Nayak, 1971, 1973) are also used in surface roughness characterization (Greenwood \& Williamson, 1966; Nayak, 1973) and to model the elastic-plastic contact of isotropic and anistropic solid bodies (McCool, 1986). The question is that the determination of $\sigma, \sigma^{\prime}$ and $\sigma^{\prime \prime}$ depends on the sample size, instrument resolution, and experimental filter used to acquire the topography data (Yan \& Komvopoulos, 1998), that is whether the rms parameters vary with the statistical sample size or the instrument resolution. Given a rough surface, an instrument with resolution $\tau$ will measure the surface height of points that are separated by a distance $\tau$. If $\tau$ is reduced, new locations on the surface are accessed. Due to the multiple scales of roughness present, a reduction in $\tau$ makes the measured profile look different for the same surface. It is thus necessary to obtain some scale-independent techniques for roughness characterization.

\subsubsection{Fractal techniques}

It is found that the power spectra of engineering surfaces produced by random processes, such as cleavage, solidification, vapour deposition, and directionally unbiased machining, have been obaserved to follow inverse power laws over a wide range of length scales (Majumdar \& Tien, 1990). This is an inherent property of fractal geometry illustrating its potential to represent surface features from the microscale down to the nanoscale (Yan \& Komvopoulos, 1998). Fractal geometry, pioneered by Manderbrot (Mandelbrot, 1967) when studying the problem of the length of Britain coastline, can be observed in various natural phenomena, such as precipitation, turbulence, and surface topography, and is characterized by continuity, nondifferentiablity, and self-affinity. Recent works (Kardar et al., 1986; Gagnepain, 1986; Majumdar \& Bhushan, 1990) have shown that the fractal geometry can be utilized to develop a scale-independent characterization technique of the fractallike behavior for a rough surface. The mathematical properties of fractal geometry can be satified by the Weierstrass-Mandelbrot (W-M) function given by (Berry \& Lewis, 1980)

$$
w(x)=\sum \gamma^{(D-2) n}\left(1-e^{i \gamma^{n} x}\right) e^{i \phi_{n}}
$$

where $w$ is a complex function of the real variable $x$. A fractal profile $z(x)$ can be obtained as the real part of $w(x)$ 


$$
\begin{aligned}
z(x) & =\operatorname{Re}[z(x)] \\
& =\sum_{n=-\infty}^{\infty} \gamma^{(D-2) n}\left[\cos \phi_{n}-\cos \left(\gamma^{n}+\phi_{n}\right)\right]
\end{aligned}
$$

where $D(1<D<2)$ is the fractal dimension of the profile, is a frequency index, $\phi_{n}$ is a random phase, and $\gamma(\gamma>1)$ is a parameter that determines the density of frequencies in the profile, which is often chosen to be 1.5. The right hand side of Equation is a superposition of cosine function with geometrically increasing frequencies. The random phase $\phi_{n}$ is used to prevent the surface profile. The approximate continuous power spectrum, $P(\omega)$, of the profile $z(x)$ given be Equation

$$
P(\omega)=\frac{1}{\omega^{(5-2 D)} \ln \gamma}
$$

is an inverse power function of the spatial frequency, $\omega$, and has been observed to hold for many engineering surfaces.

The two-variable function developed by Ausloos and Berman (Ausloos \& Berman, 1980) can be used to model fractal surfaces exhibiting corrugations in all directions. The height function of a fractal surface can be expressed

$$
z(\rho, \theta)=\left(\frac{\ln \gamma}{M}\right)^{\frac{1}{2}} \sum_{m=1}^{M} A_{m} \sum_{n=-\infty}^{\infty}\left(\kappa \gamma^{n}\right)^{(D-3)} \cdot\left\{\cos \phi_{m, n}-\cos \left[\kappa \gamma^{n} \rho \cos \left(\theta-\alpha_{m}\right)+\phi_{m, n}\right]\right\}
$$

where $D(2<D<3)$ is the fractal dimension of the surface. The physical significance of $D$ is the extent of space occupied by the rough surface, with larger $D$ values corresponding to denser profiles. For isotropic surfaces, the value of $D$ can be determined from the slope of the log-log plot of power spectrum (Wang \& Komvopoulos, 1994; Gagnepain \& RoguesCarmes, 1986). The parameter $M$ denotes the number of supposed ridges used to construct the surface. The anisotropy of the surface geometry is controlled by the magnitude of $A_{m}$. For isotropic surface, $A_{m}=A$ for all $m$ values; for anisotropic surfaces, $A_{m}$ varies with $m$. The arbitrary angle $\alpha_{m}$ is used to offset the ridges in the azimuthal direction. The parameter $\kappa$ is a wave number related to the sample size, $\kappa=\pi m / M$. The frequency index $n$ is a finite value. The lowest frequency of index $n_{\min }$ is equal to $1 / L$ and it can also be set equal to zero. The upper limit of $n$ is

$$
n_{\max }=\operatorname{int}\left[\frac{\log \left(L / L_{s}\right)}{\log \gamma}\right]
$$

where int $[. .$.$] denotes the maximum integer value of the number in the brackets, L_{s}$ is the cut-off length of sample.

By introducing a new length parameter $G$ such that $G$, the surface height function of 3D isotropic surfaces can be obtained 


$$
z(x, y)=L\left(\frac{G}{L}\right)^{D-2}\left(\frac{\ln \gamma}{M}\right)^{\frac{1}{2}} \sum_{m=1}^{M} \sum_{n=0}^{n_{\max }} \gamma^{(D-3) n} \cdot\left\{\cos \phi_{m, n}-\cos \left[\frac{2 \Pi \gamma^{n}\left(x^{2}+y^{2}\right)^{\frac{1}{2}}}{L} \cdot \cos \left(\tan ^{-1}\left(\frac{y}{x}\right)-\frac{\Pi m}{M}\right)+\phi_{m, n}\right]\right\}
$$

The Equation (11) can be used to represent a 3D isotropic fractal surface. This function of surface height provides a deterministic means of generating stochastic rough surfaces. The only unknown variables in Equation (11) are the scale dependent fractal parameters $G$ and $D$, which can be determined experimentally. Therefore, this fractal approach has the inherent capability of representing surfaces at various length scales, different from those at which the measurements were made (Yan \& Komvopoulos, 1998).

\section{Surface forces and adhesion mechanics}

Surface microstructures typically range from 0.1 to several $\mu \mathrm{m}$ in thickness with lateral dimensions of $10-500 \mu \mathrm{m}$, and lateral and vertical gaps to other structures or to the substrate of around $1 \mu \mathrm{m}$ (Maboudian \& Howe, 1997). The large surface area and small offset from adjacent surfaces makes these microstructures especially vulnerable to adhesion upon contact. The causes of strong adhesion can be traced to the interfacial forces existing at the dimensions of microstructures. These include capillary, electrostatic, van der Waals, and chemical forces.

\subsection{Surface forces and adhesion work}

There are a wide variety of surface forces (Israelachvili, 1992). Capillary, electrostatic and van der Waals forces can each contribute to adhesion under different circumstances in MEMS devices.

\subsection{1 van der Waals forces}

Van der Waals force is the force acting between atoms or small molecules, which includes dispersion force, Debye force and dipole-dipole force. The interaction potential between atoms or molecules of each force is a function of $1 / r^{6}$, which $r$ is the separation between atoms. For two flat parallel surfaces, and for separations less than a characteristic distance, $r_{0} \approx 20 \mathrm{~nm}$ (nonretarded regime), the attractive force per unit area is given by (Israelachvili, 1985)

$$
F_{v d W}(r)=\frac{A}{6 \pi r^{3}}
$$

where $A$ is the Hamaker constant to reflect the strength of the van der Waals interaction for two bodies in medium. However, for separations larger than $r_{0}$, the attraction is retarded. Taking retardation into account, it is proposed (Cheng \& Cole, 1988) that the van der Waals force per unit area is represented more accurately as

$$
F_{v d W}(r)=\frac{A}{6 \pi r^{3}} \frac{r_{0}}{r^{3}\left(r+r_{0}\right)}
$$


The work of adhesion between two surfaces interacting with each other via van der Waals interaction can be obtained by integration from contact, $r_{0, v d W}$, to infinity (Maboudian \& Howe, 1997)

$$
W_{v d W}(r)=\frac{A}{12 \pi r_{0, v d W}^{2}}
$$

\subsubsection{Electrostatic forces}

Electrostatic forces are the forces between charged bodies. Charges are known to accumulate from the ambient and migrate across insulating surfaces on silicon chips. Early in the development of integrated-circuit (IC) technology, charge migration was the source of device instabilities. Transport of both positive and negative ionic species has been observed in the presence of lateral electrical fields (Shockley, 1964). Electrostatic attraction may also arise due to a difference in the work function of the approaching surfaces. Neglecting the internal space charge regions, the force per unit area acting between surfaces with potential difference $V$ separated by an air gap with permittivity $\varepsilon_{0}$ is given by

$$
F_{e l}(r)=\frac{\varepsilon_{0} V^{2}}{2 r^{2}}
$$

and the associated energy is given by

$$
W_{e l}(r)=\frac{\varepsilon_{0} V^{2}}{2 r}
$$

\subsubsection{Capillary force}

With the presence of a thin liquid film, such as a lubricant or adsorbed water layer at the contact interface, menisci will form around the contacting and noncontacting asperities due to surface energy effects (Israelachvili, 1985). Fig.4 (a) shows the condition that the amount of liquid film volume was large enough to immerse the rough surface. When the mating surfaces are pulled apart, meniscus will formed underneath the microstructures. Then the liquid meniscus will create a pressure because of the pressure difference across the curved liquid-air interface, which is called the capillary pressure (Laplace pressure), and is given by

$$
P_{l}=\gamma_{l}\left(\frac{1}{r_{1}}+\frac{1}{r_{2}}\right)
$$

The liquid surface tension is denoted by $\gamma_{l}$, and the two radii of curvature of the liquid surface are termed by $r_{1}$ parallel to the surface normal of the substrate and $r_{2}$ (in the plane of the substrate) (Adamson, 1990; Israelachvili,1985). Since in micromechanical structures lateral dimensions are often much larger than the vertical spacing, $r_{2} \gg r_{1}$, and in this case, Equation (17) simplifies to

$$
P_{l}=\frac{\gamma_{l}}{d}\left(\cos \theta_{1}+\cos \theta_{2}\right)
$$


where $\theta_{1}, \theta_{2}$ is the upper and lower contact angle of liquid bridge, and $d$ is the separation distance between the two surfaces, equal to $r_{1}\left(\cos \theta_{1}+\cos \theta_{2}\right)$. On a hydrophilic surface $\left(\theta<90^{\circ}\right)$, such as the native oxide of silicon, the meniscus shape will be concave underneath a structure shown in Fig.4 (a). This creates an attractive capillary force that may sufficiently strong to pull the compliant structures into contact.

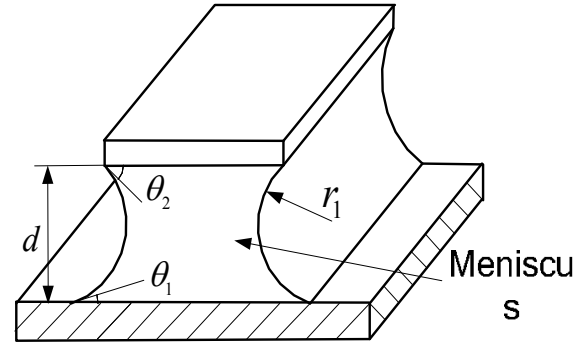

(a)

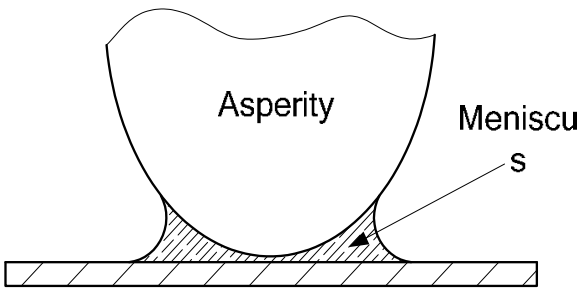

(b)

Fig. 4. Wetting and contact angle

The isolated micromenisci would occur at the contact interface, if the amount of meniscus volume were not large enough to immerse all asperities of the rough surface, as shown in Fig.4 (b), the meniscus radius at equilibrium is equal to the so-called Kelvin radius $r_{k}$. It is related to the Kelvin equation value and controlled by the relative vapor pressure relative humidity (Admoson, 1990). At equilibrium, the meniscus curvature is related to the relative vapor pressure $\left(H_{r}\right)$ by the Kelvin equation:

$$
\gamma_{l}\left(\frac{1}{r_{1}}+\frac{1}{r_{2}}\right)^{-1} \equiv r_{k}=\frac{\gamma_{l} v}{R T \log H_{r}}
$$

where $r_{1}$ and $r_{2}$ are the two radii of curvature of the meniscus, $r_{k}$ is the Kelvin radius, $\gamma_{l}$ is the surface tension of the liquid, and $v$ is its molar volume $\left(\gamma_{l} v / R T \approx 0.54 \mathrm{~nm}\right.$ for water at $20^{\circ} \mathrm{C}$ ). As two hydrophilic surfaces approach each other in a humid environment, the liquid undergoes capillary condensation as soon as the separation equals

$$
d_{0} \approx r_{k}\left(\cos \theta_{1}+\cos \theta_{2}\right)
$$

If, after the condensation has occurred, the two surfaces are pulled apart, the volume of the condensate is essentially constant and is given by $V=S_{w} d_{0}$, where $S_{w}$ is the wetted surface area (Maboudian \& Howe,1997). The effect of a liquid condensate on the adhesion force per unit area between two parallel plates is then given by

$$
F_{c a p}(d)=\frac{\gamma_{l} d_{0}}{d^{2}}\left(\cos \theta_{1}+\cos \theta_{2}\right)
$$


If we assume that, as the two surfaces are pulled apart, the meniscus breaks at a separation much larger than $d_{0}$, then integrating Equation (21) from $d_{0}$ to infinity yields the work of adhesion due to capillary forces

$$
W_{\text {cap }}=\gamma_{l}\left(\cos \theta_{1}+\cos \theta_{2}\right)
$$

\subsection{Adhesion models of single asperity}

To better understand the interfacial adhesion of MEMS devices, it is important to provide an adequate background of the prior work performed in the area of adhesive rough surface contact. For determining the interfacial adhesive behavior, several solutions have been developed and many of these theories idealize the asperity in contact with a half rigid flat as a spherical shape.

Hertz theory is the famous continuum contact mode to predict the contact area for various geometries. It relates the radius of the circle of contact $a_{\mathrm{H}}$ to the load $P$, the spherical indenter radius $R$, and the equivalent elastic modulus of the contacting materials $K$ by:

$$
P_{\mathrm{H}}=\frac{K a_{\mathrm{H}}^{3}}{R}
$$

and between the contact radius $a_{\mathrm{H}}$ and the indentation depth $\delta$,

$$
\delta=\frac{a_{\mathrm{H}}^{2}}{R}
$$

In the presence of surface forces, Hertz theory can underestimate the contact area, especially when the load diminishes to zero. Considering the contact between a rigid sphere with half rigid flat, the adhesion force $P_{a}$, between then is given be Bradely theory (Bradley, 1932) as

$$
P_{a}=2 \pi \omega R
$$

DMT theory was then proposed by Derjaguin, Muller and Toporov to account for the longranged attraction around the periphery of the contact area. The DMT model gives the contact radius $a_{D M T}$ related to the work of adhesion, $\omega$, by

$$
\begin{gathered}
P_{\mathrm{DMT}}=\frac{K a_{\mathrm{DMT}}^{3}}{R}-2 \pi \omega R \\
\delta=\frac{a_{\mathrm{DMT}}^{2}}{R}
\end{gathered}
$$

It is apparent that DMT is Hertz with an offset due to surface forces. Therefore, DMT theory applies to rigid systems, low adhesion and small radii of curvature. JKR theory, described by Johnson, Kendall and Roberts, takes the short-ranged attractive forces among the contact area into account. It related the contact radius, $a_{J K R}$, to the work of adhesion, $\omega$, as

$$
P_{\mathrm{JKR}}=\frac{K a_{\mathrm{JKR}}^{3}}{R}-\sqrt{6 \pi \omega K a_{\mathrm{JKR}}^{3}}
$$




$$
\delta=\frac{a_{\mathrm{JKR}}^{2}}{R}-\frac{2}{3} \sqrt{\frac{6 \pi \omega a_{\mathrm{JKR}}^{3}}{K}}
$$

JKR theory applies well to highly adhesive systems that have large radii of curvature and low stiffness. To bridge the DMT theory and JKR theory, by following the analysis of Tabor (Tabor, 1977; Muller et al., 1980) pointed out that the two theories represented the opposite extremes of a dimensionless parameter $\mu$ given as

$$
\mu=\left(\frac{R \omega^{2}}{E^{\prime 2} \varepsilon^{2}}\right)
$$

where $\varepsilon$ is the equilibrium spacing in the lennard-Jones potential. $\mu$ can be interpreted as the ratio of elastic deformation resulting from adhesion to the effective range of surface forces.

A more complex, yet more accurate, description of sphere-flat adhesion mechanic, which is referred as MD model, was formulated by Maugis (Maugis, 1992). By analogy with the plastic zone ahead of a crack, the adhesion is represented by a constant additive traction acting over an annular region around the contact area. The ratio of the width of the annular region to the radius of the contact area is denoted by $m$. The set of equations relating the dimensionless load, approach is

$$
\begin{gathered}
1=\frac{\lambda A^{2}}{2}\left[\sqrt{m^{2}-1}+\left(m^{2}-2\right) \operatorname{tg}^{-1} \sqrt{m^{2}-1}\right]+\frac{4 \lambda^{2} A}{3}\left[1-m+\sqrt{m^{2}-1} t^{-1} \sqrt{m^{2}-1}\right] \\
\bar{P}=A^{3}-\lambda A^{2}\left(\sqrt{m^{2}-1}+m^{2} \operatorname{tg}^{-1} \sqrt{m^{2}-1}\right) \\
\Delta=A^{2}-\frac{4 \lambda A}{3} \sqrt{m^{2}-1}
\end{gathered}
$$

where $\lambda$ is another dimensionless number, called transition parameter $\lambda$, and is related to $\mu$ by $\lambda=1.157 \mu$. The dimensionless parameters that appear in the above equations are defined as follows: $\lambda=\frac{2 \sigma_{0}}{\left(\pi \omega K^{2} / R\right)^{1 / 3}}, \bar{P}=\frac{P}{\pi \omega R}, A=\frac{a_{M D}}{\left(\pi \omega R^{2} / K\right)^{1 / 3}}, \Delta=\frac{\delta}{\left(\pi \omega^{2} R / K^{2}\right)^{1 / 3}}$, where the adhesion work $\omega$ is defined as $\sigma_{0} h_{0}, \sigma_{0}$ is the adhesive attraction equals Dugdale stress and $h_{0}$ is the effective range of Dugdale stress.

For each previous mentioned theories were presented, there may be cases when assumptions made for a given approach do not exactly describe the materials combinations or the geometry, which are depicted physically in Fig.5.

Following the analysis of Maguis, Kim et al (Kim et al., 1998) offers an extension of the MD solution by adding to the solution regime when the asperity might not be in physical contact but still in the range of adhesion. The KMJ extensions explained by Kim is

$$
1=\frac{\pi}{4} \lambda c^{2}+\frac{2}{3} c(\pi-2) \lambda^{2}+\xi
$$




$$
\begin{gathered}
\bar{P}=-\frac{\pi \lambda c^{2}}{2} \\
\Delta=-\frac{4 C \lambda}{3}-\frac{2}{\pi} \frac{\xi}{\lambda}
\end{gathered}
$$

where $C=\frac{c}{\left(\pi \omega R^{2} / K\right)^{1 / 3}}, c$ represents the adhesive contact zone radius, $\xi$ is ratio of $h_{g} / h_{0}$, and $h_{g}, h_{0}$ are the gap between the deformed asperity at $r=0$ and $r=c$ respectively shown in Fig.5(e.)

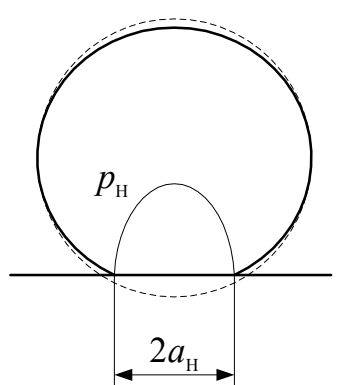

(a). Hertz

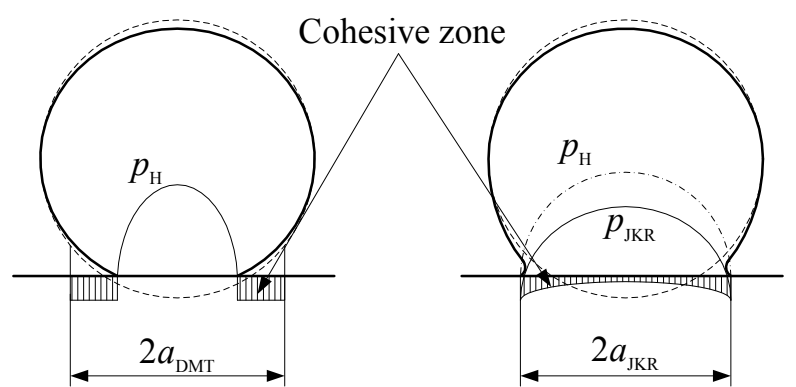

(b). DMT

(c). JKR

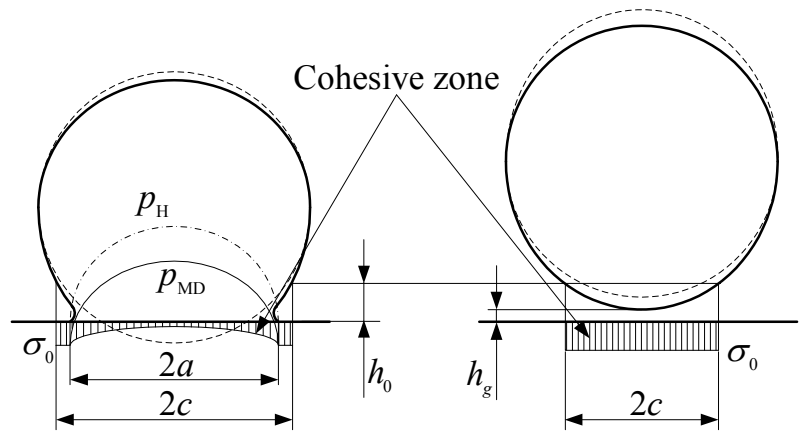

(d). MD

(e). KMJ

Fig. 5. Interactive forces for the (a). Hertz, (b). DMT, (c). JKR, (d). MD, and (e).KMJ.

\subsection{Adhesion for micro-sized rough surface}

In practice, the contact at the surface interface is governed by asperity interaction. Since the surfaces are not smooth, contact of two multiscale rough surfaces will occur only at discrete points which sustain the total compressive force. The typical contact interface which is formed of contact spots of different sizes that are spatially distributed randomly over the interface. The size of contact spots ranges from nanometers to micrometers, making adhesion a multiscale phenomenon. 


\subsubsection{Statistical adhesion theories for micro-sized rough surface}

Most contact theories of the rough surface thus far are mostly based upon the conventional statistical parameters such as standard deviation of asperity heights, slope and radius of curvature (Greenwood \& Williamson, 1966; Fuller \& Tabor, 1975). The GreenwoodWilliamson (GW) model assumes the surface to be composed of hemispherical asperities all having the same radius of curvature $R$. The summit heights or asperity peaks are distributed randomly about a mean summit plane and follow a Gaussian distribution with a standard deviation, $\sigma$. If there exists a probability density function $\varphi(z)$ of asperity heights, then it is possible to find the probability that an asperity will be greater than a certain height, $d$. The distance $d$ represents the length from the mean plane of asperity heights to the smooth surface. The probability that an asperity height is greater than $d$ is given by:

$$
\int_{d}^{\infty} \varphi(z) \mathrm{d} z
$$

Therefore, it follows that the number of asperities in contact is represented by

$$
n=N \int_{d}^{\infty} \varphi(z) \mathrm{d} z
$$

where $N$ represents the total number of asperities.

Having the numerical expressions for the non-dimensional contact radius $\left(A_{i}\right)$ and load $\left(\bar{P}_{i}\right)$ for a single asperity as a function of $\Delta$, the total contact area and load can be formed (Morrow et al., 2003)

$$
\begin{gathered}
A_{\text {total }}=N\left(\frac{\pi \omega R^{2}}{K}\right)^{2 / 3} \int_{d}^{\infty} \pi A_{i}(\Delta)^{2} \varphi(z) \mathrm{d} z \\
P_{\text {total }}=N \pi \omega R \int_{d}^{\infty} \bar{P}_{i}(\Delta)^{2} \varphi(z) \mathrm{d} z
\end{gathered}
$$

The asperities that have a height than $d$ greater than are deformed by a distance $\delta=z-d$. Assuming a Gaussian distribution and to have a relationship between $\Delta$ and $z$, the following equation can be obtained:

$$
\frac{P_{\text {total }}}{N \pi \omega R P_{c}(\lambda)}=\int_{d}^{\infty} \bar{P}_{i}\left(\frac{\delta}{\bar{\delta}}\right)^{2} \exp \left(-\frac{(\delta+d)^{2}}{2 \sigma^{2}}\right) \mathrm{d} \delta
$$

where $\bar{\delta}$ is $\left(\pi^{2} \omega^{2} R / K^{2}\right)^{1 / 3}$. The above equation is only valid when the smooth surface progressively approaches the rough surface until a minimum $d$ is reached [Fuller and Tabor]. Because of the existence of $\delta_{c}\left(-\left(3 \pi^{2} \omega^{2} R / 4 K^{2}\right)^{1 / 3}\right)$ to abrupt rupture, or pull-off, the asperities will no longer contribute to the adherence force when asperities were extended above $\delta_{c}$. Therefore, Morrow et al made the adjustment of the lower integration limit by the amount $\delta_{c}(\lambda)$, the adhesion model given above then takes the form: 


$$
\begin{gathered}
\frac{P_{\text {total }}}{N \pi \omega R P_{c}(\lambda)}=\frac{1}{P_{c}(\lambda) \sqrt{2 \pi}} \int_{-2 \delta_{c}^{*}(\lambda)}^{\infty} \bar{P}_{i}\left(\frac{\delta}{\bar{\delta}}\right)^{2} \exp \left(-\frac{\left(\delta^{*}+d^{*}\right)^{2}}{2}\right) \mathrm{d} \delta^{*} \\
\frac{A_{\text {total }}}{N\left(\pi \omega R^{2} / K\right)^{2 / 3} A_{c}(\lambda)}=\frac{\pi}{A_{c}(\lambda) \sqrt{2 \pi}} \int_{-2 \delta_{c}^{*}(\lambda)}^{\infty} A\left(\frac{\delta^{*}}{\bar{\delta}^{*}}\right)^{2} \exp \left(-\frac{\left(\delta^{*}+d^{*}\right)^{2}}{2}\right) \mathrm{d} \delta^{*}
\end{gathered}
$$

Any term in above equation that has a superscript has been divided by $\sigma$. The improvement equation by Morrow is similar in form to the rough surface integral of Fuller and Tabor, but has some important difference. The most important is that the lower integration limit $\delta_{c}$ and $P_{c}$ are functions of $\lambda$, which gives the solution validity over the entire range of the transition parameter. $P_{c}$ represents the force at which the system becomes stable under force control. The normalization factor $P_{c}(\lambda)$ is determined by finding the point at which the tangent for load deflection curve becomes zero. $\delta_{c}(\lambda)$ can also be determined in a similar manner. The critical step in obtaining an adhesive rough surface solution is to find the load at which the system becomes unstable, i. e. the minimum pull-off force $\left(P_{\min }\right)$. The $d^{*}$ can be solved by set the derivative of Eq. (42) equals to zero (Morrow, 2003).

\subsubsection{Fractal adhesion theories for micro-sized rough surface}

The statistically based adhesive theory can be used with confidence as long as the length scale is known before hand (Morrow, 2003). It is well documented that surfaces exhibit roughness on many different length scales (Majumdar \& Bhushan, 1990; Majumdar, 1989; Majumdar \& Bhushan, 1991). The topography of any surface can be thought of as roughness surperimposed on top of roughness. Majumdar et al (Majumdar \& Bhushan, 1990; Majumdar \& Tien, 1990) have proven that the multi-scale nature for surface roughness can be represented by fractal geometry. Then it is reasonable to establish the adhesion model of rough interface based on the fractal parameters. Majumdar (Majumdar \& Bhushan, 1991) has argued that the size distribution of contact spots can be given as:

$$
n(s)=\frac{D}{2 s}\left(\frac{s_{l}}{s}\right)^{D / 2}
$$

where $s$ is the contact area and $s_{l}$ represents the largest spot contact area.

\subsubsection{Fractal model for adhesive contact of JKR type}

By assuming that the plasticity plays a minor role in the asperity contact due to the light loading conditions, Morrow et al proposed a fractal model for adhesive contact of JKR type (Morrow \& Lovell, 2003). The model follows the example set forth by Majumdar and Bhushan (Majumdar \& Bhushan, 1991). It was assumed that the interference which a spherical has with a rigid plane is given by:

$$
\delta=G^{(D-1)} l^{(2-D)}
$$

where $l$ is the length scale of the asperity as shown in Fig. 6. 
A relationship between the truncated and real contact area is developed by equaling the interference distance for both the Hertizan truncated and JKR conditions:

$$
s_{J K R}=9.25\left(\frac{s^{\prime D} G^{2(1-D)} \omega}{K}\right)^{2 / 3}\left(1.68-0.61 \sqrt{5.95-\frac{\sqrt{\left(s^{\prime} \pi\right) / 2}}{\left(\left(s^{\prime D} G^{2(1-D)} \omega\right) / K\right)^{1 / 3}}}\right)
$$

Following the work of Majumdar and Bhushan, the expression for the interference, $\delta$, in terms of truncated area $s^{\prime}$ :

$$
\delta=G^{(D-1)} \mathcal{S}^{(2-D) / 2}
$$

The radius of curvature at the asperity tip in terms of fractal parameters (Majumdar \& Bhusha, 1991)

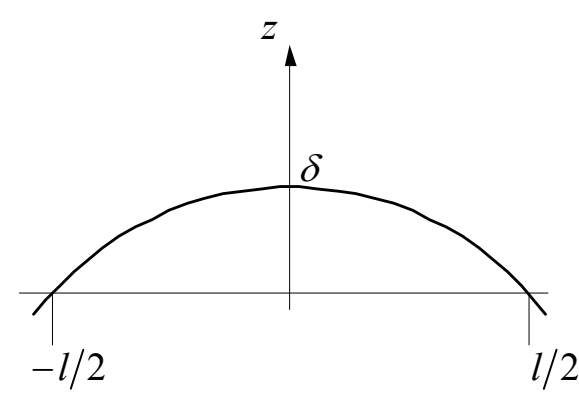

(a) Geometry of contact spot with a given interference

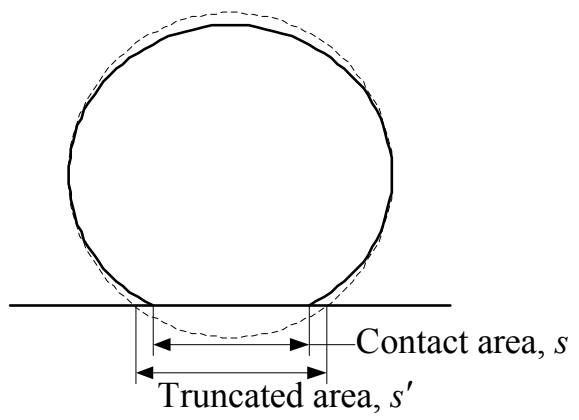

(b) Truncated area and contact area

Fig. 6. Fractal approximation of asperity contact (Morrow, 2003)

$$
R=\frac{s^{\prime D / 2}}{\pi^{2} G^{D-1}}
$$

Because of the surface attraction, the asperities are stretched when the contacting surfaces are pulled away. The critical interference $\delta_{c}$ is given

$$
\delta_{c}=-\left(\frac{3 \omega^{2} s^{\prime D / 2}}{4 K^{2} G^{D-1}}\right)^{1 / 3}
$$

The critical contact area $s_{c}^{\prime}$ for adhesion is broken is:

$$
s_{c}^{\prime}=\left(\frac{3 \omega^{2}}{4 K^{2}}\right)^{1 /(3-2 D)} G^{4(1-D) /(3-2 D)}
$$

Supposing that the size distribution of the asperities, $n(s)$, is known, the real area of contact can be integrated 


$$
s_{\text {real }}^{\prime}=\int_{s_{c}^{\prime}}^{s_{l}^{\prime}} S^{\prime} n(s) \mathrm{d} s^{\prime}=\frac{D}{2-D}\left(s_{l}^{\prime}-s_{c}^{\prime 1-D / 2} s_{l}^{\prime D / 2}\right)
$$

where $s_{l}^{\prime}$ represents the truncated area of the largest contact spot and is related with the total truncated area $S^{\prime}$ with

$$
s_{l}^{\prime}=\left(\frac{3-D}{D-1}\right) S^{\prime}
$$

Then the pull-off force is

$$
P=\int_{s_{c}^{\prime}}^{s_{l}^{\prime}} P\left(s^{\prime}\right) n(s) \mathrm{d} s^{\prime}
$$

The above Equation can be numerically integrated and solved (Morrow, 2003).

\subsubsection{Fractal elastic-adhesive model}

Based on the work of Yan and Komvopolous (Yan \& Komvopoulos, 1998), Morrow proposed a 3D fractal elastic adhesive rough surface solution methodology (Morrow, 2003). In order to develop easy to use expressions for the asperity interference, Yan and Komvopoulos developed a two-dimensional form of Equation (11) to account for the $M$ number of ridges by introducing a multiplicative factor which was eventually set equal to 1 . This implied that a two-dimensional W-M function could be used to approximate a fractal function in three dimensions. Yan states in (Yan \& Komvopoulos, 1998) that since the radius of curvature of each asperity is much greater than the height of the asperity then this relationship can be assumed to be

$$
a^{\prime}=2 R \delta
$$

where $a^{\prime}$ is the truncated contact radius. Then the radius of curvature is:

$$
R=\frac{s^{\prime(D-1) / 2}}{2^{5-D} \pi^{(D-1) / 2} G^{D-2}(\ln \gamma)^{1 / 2}}
$$

where the truncated contact area $s^{\prime}$ can be expressed as

$$
s^{\prime}=\left(\frac{\delta}{2^{4-D} \pi^{(D-3) / 2} G^{D-2}(\ln \gamma)^{1 / 2}}\right)^{2 /(3-D)}
$$

The fractal relationship for the radius of curvature, $R$ can be used to modify the transition parameter $\lambda$ which is related to fractal dimensions

$$
\lambda=1.16\left(\frac{\omega s^{(D-1) / 2}}{2^{5-D} K^{2} \pi^{(D-1) / 2} G^{D-2} \sqrt{\ln \eta} z_{0}^{3}}\right)
$$

where $z_{0}$ is the intermolecular distance. From the fractal adhesion model, it can be found that transition parameter $\lambda$ is no more a constant, but a variant with the approaching 
distance $\delta$ of the asperity during the adhesive contact process. Then the truncated area for each $i$ th asperity can be determined based on a given interference $\delta_{i}$ :

$$
\frac{s_{i}^{\prime}}{\pi}=2\left(\frac{s^{\prime(D-1) / 2}}{2^{5-D} \pi^{(D-1) / 2} G^{D-2}(\ln \gamma)^{1 / 2}}\right) \delta_{i}
$$

Equation 58 can be solved using a fixed point iteration scheme to determine the truncated area. It should be noted that the truncated area is used for the first iteration of the algorithm only. To solve for the total load and contact area, Morrow proposed a novel numerical algorithm. In this algorithm the Maugis-Dugdale solution is used to model the micro-sized contact of each asperity. The first step is to generate the surface topography from the fractal parameters using W-M function. Next the surface is offset to introduce an initial penetration into the rigid plane. The interference, $\delta_{i}$, of each asperity is determined. Based on this interference, the truncated area is subsequently computed for only the asperities that are physically interfering with the rigid plane and is then used to compute the radius of curvature and transition parameter $\lambda$ for each asperity. Once the truncated area is initially determined, the main iteration scheme is started to determine the real area of contact for each asperity. Using the values for $\lambda$ and $R$, the contact radius, $m$ and $\delta_{\text {com }}$ are computed. These values are computed based on the adhesive contact solution of Maugis given in Equations (31-33). Once the iterations have converged, the values of the load, $P_{i}$, and area, $S_{i}$ are added to the totals $\left(P_{\text {total }}\right.$ and $\left.S_{\text {total }}\right)$. All interfering asperities are iterated on in this fashion and then the surface is moved to the next separation locatioln and the procedure starts once again.

\subsubsection{Fractal elastic-plastic adhesion model}

According to Majumdar-Bhushan model (Majumdar \& Bhushan, 1991), the truncated area $s^{\prime}>s_{c}^{\prime}$ are elastically deformed since they satisfy the condition of $\delta<\delta_{c}$, whereas asperities with $s^{\prime} \leq s_{c}^{\prime}$ satisfy the plastic flow criterion and are thus considered to be in fully plastic deformation state. This result is in disagreement with that derived from the GW model. The reason for this disagreement is that the present analysis accounts for the dependence of the curvature radius on microcontact area, whereas in the GW model the curvature radius of asperity is considered to be invariant. The critical microcontact area for plastic flow of the entire asperity is

$$
s_{c}^{\prime}=\left(\frac{2^{11-2 D}}{9 \pi^{4-D}} G^{2 D-4} \ln \gamma\left(\frac{E}{H}\right)^{2}\right)^{1 /(D-2)}
$$

The elastic adhesion of asperities can be analyzed by Morrow method (Morrow, 2003). Assuming the asperity is at a fully plastic microcontact, the contact pressure within the contact zone is the hardness, $H$. Then with the Maugis-Dugdale approximation to the adhesive interaction, the adhesive contact pressure for the microcontact can be approximated as (Peng \& Guo, 2007)

$$
p_{p}=\left\{\begin{array}{cc}
H-\frac{2 \sigma_{0}}{\pi} \tan ^{-1} \sqrt{\frac{c^{2}-a^{2}}{a^{2}-x^{2}}} & |x| \ll a \\
-\sigma_{0} & a<|x|<c
\end{array}\right.
$$


The adherence force can be obtained by integration over the contact and cohesive zones

$$
P_{p}=H s-2 \sigma_{0} a^{2}\left[\sqrt{\frac{c^{2}}{a^{2}}-1}+\frac{c^{2}}{a^{2}} \tan ^{-1} \sqrt{\frac{c^{2}}{a^{2}}-1}\right]
$$

where $s=2 \pi a^{2}$ is the real contact area. When plastically deformed, the contact area $s$ of the microcontact is just the truncated area, which means $a=a^{\prime}$ and is

$$
a=\frac{1}{2}\left(\frac{\delta}{2 G^{D-2}(\ln \gamma)^{1 / 2}}\right)^{1 /(3-D)}
$$

The radius of cohesive zone $c$ can also be determined by geometrical consideration

$$
c=\frac{1}{2}\left(\frac{\delta+h_{0}}{2 G^{D-2}(\ln \gamma)^{1 / 2}}\right)^{1 /(3-D)}
$$

If $s_{l}^{\prime}>s_{c}^{\prime}$, both elastic and fully plastic microcontacts exists. Thus the total adherence force for the fractal surface includes the elastic adhesion and plastic adhesion forces, which is

$$
F_{t}=\sum_{j} P_{e j}+\sum_{j} P_{p j}
$$

where $P_{e j}$ is the adherence force of $j$ th asperity in elastic contact which can be determined by MD theory utilizing the Morrow method, and $P_{p j}$ is the adherence force of $j$ th asperity in plastic contact which can be calculated by Equation (61).

\subsubsection{Adhesion model for meniscus stiction}

The issue of meniscus force is of critical importance for the microsized interfacial interaction mechanism, such as in the magnetic storage hard-disk drives (Bhushan, 1996). For a given interacting system and environmental parameters, the Laplace pressure and the meniscus height can be assumed to be a constant, whose character is similar to that of the Dugdale stress in linear elastic fracture mechanics. To solve the adhesive problem of the capillary force due to meniscus, the effective work of adhesion can be defined by the product of the meniscus height and Laplace pressure (Xue \& Polycarpou,200; Peng et al., 2009)

$$
\omega_{l}=h_{c} p_{l}
$$

Then by substituting the $\sigma_{0}$ with $p_{l}$, and $h_{0}$ with $h_{c}$, the above methodology, mentioned in section 3.3.2.2 and 3.3.2.3, can be adopted to solve the sitiction problem in presence of meniscus.

\section{Summary}

In this chapter, we have attempted to present a description of issues and techniques in the interfacial adhesion for the MEMS devices. We firstly discus the complexity of the surface 
microstructure. Then we present the techniques to characterize the micro-scale surfaces. Finally, we introduce the adhesion models to interpret the adhesive interaction of MEMS devices.

It is hoped that the introductions in this chapter can gain the rational understanding leading to the design of better MEMS structures in the technologically field. The interpretation of interfacial adhesion is challenging for the application of MEMS technology. It is further complicated by the inability to observe the interfacial interactions directly, resulting in conclusions from inference. The gap between theoretical research of rough surface adhesion and the real world where thousands or millions of asperities are involved remains enormous. Then it is clear that there is still a great deal of research necessary to obtain a comprehensive understanding of adhesion at the microscale. The high-resolution instrument should be developed and well calibrated, with which one can measure both the microstructure topography and adhesion, especially the biological sample and hydrophilic surface. It is essential that the proper data processing method should be presented to reflect the intrinsic characters more accurately, and helps to understand the sources of error.

\section{References}

Adamson, A. W. (1990). Physical Chemistry of Surfaces, 5th ed., Wiley, New York.

Ausloos and Berman. (1980). A multivariate Weierstrass-Mandelbrot function, Proceedings of the Royal Society A 370, 459.

Bennet, J.M. \& Dancy, J.H. (1981). Stylus profiling instrument for measuring statistical properties of smooth optical surfaces, Applied Optics Vol. 20: 1785-1802.

Berry, M. V. \& Hannay, J. H. (1978). Topography of Random Surfaces, Nature Vol. 271: 573.

Berry, M. V. \& Lewis, Z. V. (1980). On the Weierstrass-Mandelbrot Fractal Function, Proceedings of the Royal Society A Vol. 370: 459-484.

Binggeli, M., Christoph, R., Hintermann, H.E., Colchero, J., Marti, O. (1993). Friction Force Measurements on Potential Controlled Graphite in an Electrolytic Environment, Nanotechnology Vol. 4: 59-63.

Binnig, G., Quate, C. F. and Gerber, C. (1986). Atomic Force Microscope, Physics Review Letter Vol. 56: 930-933.

Binnig, G. \& Rohrer, H. (1982). Scanning tunneling microscopy, Helvetica Physica Acta Vol. 55: 726-735.

Binnig, G. \& Smith, D.P.E. (1986). Single-Tube Three-Dimensional Scanner for Scanning Tunneling Microscopy, Review of Scientific Instruments Vol. 57: 1688.

Bhushan, B. (1999). Handbook of Micro-Nanotribology, Second Edition. Bharat Bhushan. CRC Press.

Bhushan, B. (1996). Tribology and Mechanics of Magnetic Storage Devices, 2nd ed., Springer, New York.

Bhushan, B. \& Blackman, G.S. (1991). Atomic Force Microscopy of Magnetic Rigid Disks and Sliders and Its Applications to Tribology, Journal of Tribology Vol. 113: 452-458.

Bhushan, B. \& Dugger, M. T. (1990). Real Contact Area Measurements on Magnetic Rigid Disks, Wear Vol. 137: 41-50.

Bhushan, B., Sundararajan, S., Scott, W.W., and Chilamakuri, S. (1997). Stiction Analysis of Magnetic Tapes, IEEE Transactions on Magnetics Vol. 33: 3211-3213.

Bhushan, B., Wyant, J.C., Meiling, J. (1988). A new three-dimensional non-contact digital optical profiler, Wear Vol. 122: 301-312. 
Bradley, R. S. (1932). The coercive force between solid surfaces and the surface energy of solids, Philosophical Magazine Vol. 13: 853-862.

Brown, S. R. and Scholz, C. H. (1985). Closure of Random Elastic Surfaces in Contact, Journal of Geophysical Research Vol. 90: 5531-5545.

Burnham, N.A., Domiguez, D.D., Mowery, R.L., Colton, R.J. (1990). Probing the Surface Forces of Monolayer Films with an Atomic Force Microscope, Physics Review Letter Vol. 64: 1931-1934.

Chang, W. R., Etsion, I., and Bogy, D. B. (1987). An Elastic-Plastic Model for the Contact of Rough Surfaces, Journal of Tribology Vol. 109: 257-263.

Cheng, E. \& Cole, M. W. (1988). Retardation and Many Body Effects in Multilayer Film Adsorption, Physics Review B Vol. 38: 987-995.

Fang, F. Z., Xu, Z. W. and Dong, S. (2008). Study on phase images of a carbon nanotube probe in atomic force microscopy, Measurement Science \& Technology Vol. 19(No. 5) doi:10.1088/0957-0233/19/5/055501.

Fuller, K. \& Tabor, D. (1975). The effect of surface roughness on the adhesion of elastic solids, Proceedings of the Royal Society A Vol. 345: 327-342.

Gangepain, J. \& Roques-Carmes. (1986). Fractal Approach to Two-Dimensional and ThreeDimensional Surface Roughness, Wear Vol. 109: 119-126.

Greenwood, J. A. \& Williamson, J. B. P. (1966). Contact of Nominally Flat Surfaces, Proceedings of the Royal Society A Vol. 295: 300-319.

Hug, H.J., Moser, A., Jung, Th., Fritz, O., Wadas, A., Parashikor, I., Guntherodt, H.J. (1993). Low Temperature Magnetic Force Microscopy, Review of Scientific Instruments Vol. 64: 2920-2925.

Israelachvili, J. N. (1985). Intermolecular and Surface Forces. Academic, London.

Kardar, M., Parisi, G., and Zhang, Y. C. (1986). Dynamic Scaling of Growing Interfaces, Physics Review Letter Vol. 56: 889-892.

Kim, K.S., McMeeking, R.M. and Johnson. K.L. (1998). Adhesion, slip, cohesive zones and energy fluxes for elastic spheres in contact, Journal of the Mechanics and Physics of Solids Vol. 46:243-266.

Maguis, D. (1992). Adhesion of spheres: The JKR-DMT transition using a Dugdale model, Journal of Collid and Interface Science Vol. 150(No. 1): 243-269.

Maboudian, R. \& Howe. R. T. (1997). Critical Review: Adhesion in surface micromechanical structures, Journal of Vacuum Science \& Technology B Vol. 15(No.1): 1-20.

Majumdar, A. (1989). Fractal surfaces and their Applications to Surface Phenomena. PhD thesis, University of California, Berkley.

Majumdar, A. \& Bhushan, B. (1991). Fractal model of elastic-plastic contact between rough surfaces, Journal of Tribology Vol. 113:1-11.

Majumdar, A. and Bhushan, B. (1990). Role of Fractal Geometry in Roughness Characterization and Contact Mechanics of Surfaces, Journal of Tribology Vol. 112: 205-216.

Majumdar, A. \& Tien, C. L. (1990). Fractal Characterization and Simulation of Rough Surfaces, Wear Vol. 136: 313-327.

Mandelbrot, B. B. (1967). How Long is the Coast of Britain? Statistical Self-Similarity and Fractional Dimension. Science Vol. 155: 636-638.

Marti, O., Drake, B., Hansma, P.K. (1987). Atomic Force Microscopy of Liquid-Covered Surfaces: Atomic Resolution Images, Applied Physics Letter Vol. 51: 484-486. 
McCool, J. I. (1986). Comparison of Models for the Contact of Rough Surfaces, Wear Vol. 107: 37-60.

Morrow, A. (2003). Adhesive Rough Surface Contact, Ph.D. thesis, University of Pittsburgh, Pittsburgh.

Morrow, C., Lovell, M.R., Ning, X. (2003). A JKR-DMT transition solution for adhesive rough surface contact, Journal of Physics D Applied Physics Vol. 36: 534-540.

Myshkin, N.K., Ya. A., Grigoriev, S.A., Chizhik, Choi, K.Y., Petrokovets, M.I. (2003). Surface roughness and texture analysis in microscale, Wear Vol. 254: 1001-1009.

Myshkin, N.K., Ya., Grigoriev, A., Kholodilov, O.V. (1992). Quantitative analysis of surface topography using scanning electron microscopy, Wear Vol.153 (No.1): 119-133.

Muller, V. M., Yushchenko, V. S., B. V. Derjaguin. (1980). On the influence of molecular forces on the deformation of anelastic sphere and its sticking to a rigid plane, Journal of Colloid Science on Science Vol. 77: 91-101.

Nayak, P. R. (1971). Random Process Model of Rough Surfaces, Journal of Lubrication Technology Vol. 93: 398-407.

Nayak, P. R. (1973). Random Process Model of Rough Surfaces in Plastic Contact, Wear Vol. 26: 305-333.

Oden, P.I., Majumdar, A., Bhushan, B., Padmanabhan, A., and Graham, J.J. (1992). AFM Imaging, Roughness Analysis and Contact Mechanics of Magnetic Tape and Head Surfaces, Journal of Tribology Vol. 114: 666-674.

Papoulis, A. (1965). Probability, Random Variables and Stochastic Processes, McGraw Hill, New York.

Peng, Y. F. \& Guo, Y. B. (2007). An Adhesion Model for Elastic-Plastic Fractal Surfaces, Journal of Applied Physics Vol. 102(No.5): 3510-7.

Peng, Y. F., Guo, Y. B., Hong, Y. Q. (2009). An Adhesion Model for Elastic-contacting Fractal Surfaces in Presence of Meniscus, ASME Journal of Tribology Vol. 131: 024504-1-5.

Sarid, D. (1191). Scanning Force Microscopy, Oxford University Press, New York.

Sayles, R. S. and Thomas, T. R. (1978). Surface Topography as a Nonstationary Random Process, Nature Vol. 271: 431-434.

Shockley, W., Hooper, W.W., Queisser, H.J. and Schroen, W. (1964). Mobile electric charges on insulating oxides with application to oxide covered silicon p-n junctions, Surface Science Vol. 2: 277-287.

Tabor, D. (1977). Surface forces and surface interactions, Journal of Colloid Science on Science Vol. 58: 2-13.

Thomas, T. R. (1982). Rough Surfaces, Longman, New York.

Wang, S. \& Komvopoulos, K. (1994). A fractal theory of the interfacial temperature distribution in the slow sliding regime: Part I - Elastic contact and heat transfer analysis, Journal of Tribology Vol. 116: 812 - 823.

Williams, E. D. \& Bartlet, N. C. (1991). Thermodynamics of Surface Morphology, Science Vol. 251: 393-400.

Xue, X. \& Polycarpou A. (2007). An improved meniscus surface model for contacting rough surfaces, Journal of Colloid and Interface Science Vol. 311: 203-211.

Yamachika, R. et al. (2004). Controlled Atomic Doping of a Single C60 Molecule, Science Vol. 304:281-284.

Yan, W. \& Komvopoulos, K. (1998). Contact analysis of elastic-plastic fractal surfaces, Journal of Applied Physics Vol. 84: 3617. 
(C) 2012 The Author(s). Licensee IntechOpen. This is an open access article distributed under the terms of the Creative Commons Attribution 3.0 License, which permits unrestricted use, distribution, and reproduction in any medium, provided the original work is properly cited. 